\title{
Microbiology Susceptibility Subcategory
}

National Cancer Institute

\section{Source}

National Cancer Institute. Microbiology Susceptibility Subcategory. NCI Thesaurus. Code C87943.

A subdivision of microbiology susceptibility data. 